\title{
Editorial
}

\section{Statistical Modeling of Hydroclimatological Processes}

\author{
Hung Soo Kim, ${ }^{1}$ Vijay P. Singh, ${ }^{2}$ and Ji Chen ${ }^{3}$ \\ ${ }^{1}$ Department of Civil Engineering, Inha University, Incheon, Republic of Korea \\ ${ }^{2}$ Department of Biological \& Agricultural Engineering and Zachry Department of Civil Engineering, Texas A\&M University, \\ College Station, TX, USA \\ ${ }^{3}$ Department of Civil Engineering, The University of Hong Kong, Pokfulam, Hong Kong \\ Correspondence should be addressed to Hung Soo Kim; sookim@inha.ac.kr
}

Received 3 August 2016; Accepted 7 August 2016

Copyright ( $\odot 2016$ Hung Soo Kim et al. This is an open access article distributed under the Creative Commons Attribution License, which permits unrestricted use, distribution, and reproduction in any medium, provided the original work is properly cited.

The aim of this special issue is to provide a forum for advances in the analysis and assessment of heavy precipitation, floods, and droughts. This issue covers a wide range of topics, such as statistical and stochastic methods for extreme events, rainfallrunoff modeling, spatiotemporal analysis of natural disasters, predicting and adapting to floods or droughts due to climate change, applying of advanced scientific technology, and risk based design for disaster management.

Therefore, the guest editors invited contributors who are experts in the aforementioned fields and finally 9 papers were accepted. The papers cover some wide ranges of topics which treated (1) extreme climate signals in hydrometeorological processes, (2) downscaling and decomposition of rainfall data for climate change analysis, and (3) understating seasonal hydrologic mechanism, drought forecasting, and long-term simulation of streamflow using radar rainfall.

(1) M. Martinkova and M. Hanel carried out the disaggregation of precipitation into prevailing stratiform and convective component on the observational data, then analyzed trends in characteristics of disaggregated events, and assessed correlation of precipitation intensities with daily mean temperature. F. Wang and J. Niu studied stochastic dynamic variability of precipitation for upper, middle, and lower reaches of the Heihe River basin, China, and found that about 2-year significant variability in the lower reach of the river occurred and this is essentially depicted by Artic Oscillation. C. Onyutha applied five hydrological models for testing model performances using extreme events. He recommended the model can be chosen with modeling objectives.

(2) M. Saifullah et al. investigated the long-term changes in precipitation and runoff in the Yihe River basin, China. They tried to quantify the impact of precipitation and land surface change on runoff. Then they found that the reduction of annual runoff is due to precipitation variability of $56.38-67.58 \%$ and land surface change of $43.62-32.32 \%$. C. Yoo et al. evaluated the effect of climate change on daily rainfall, especially on the mean number of wet days and the mean rainfall intensity using Markov chain model decomposition of monthly rainfall into daily rainfall. L. Campozano et al. tested the performance of downscaling methods to improve the results of GCMs using the statistical downscaling model (SDSM), artificial neural networks (ANNs), and the least squares support vector machines (LS-SVM) approach.

(3) B. Pan and Z. Cong evaluated the temporal scale transition revealed by observation and simulation using information theoretical framework called Aleatory Epistemic Uncertainty Estimation and found that the flows of hydrologic terms across temporal scales were related with the catchments' seasonality type. J. Y. Shin et al. developed a probabilistic scheme for drought forecasting and outlook combined with quantification of the prediction uncertainties. They showed that the scheme can be used for forecasting drought conditions in the suggested outlook framework. H. Noh et al. tried to simulate long-term streamflow using the 
results from rain-radar for hydrologic purpose while the radar rainfall was used for short-term event and flood prediction analysis.

\section{Acknowledgments}

We would like to thank all the contributors of this special issue for their participations and efforts. We express our sincere gratitude to authors and reviewers of papers for their good paper submissions and valuable comments and discussions.

Hung Soo Kim Vijay P. Singh Ji Chen 

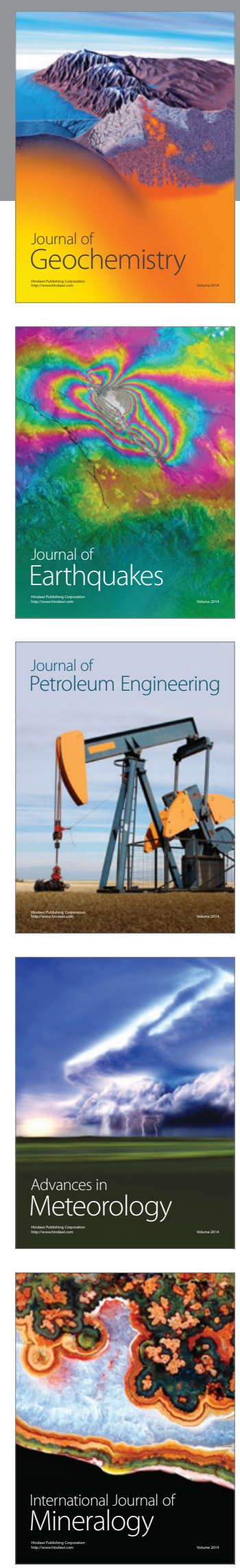
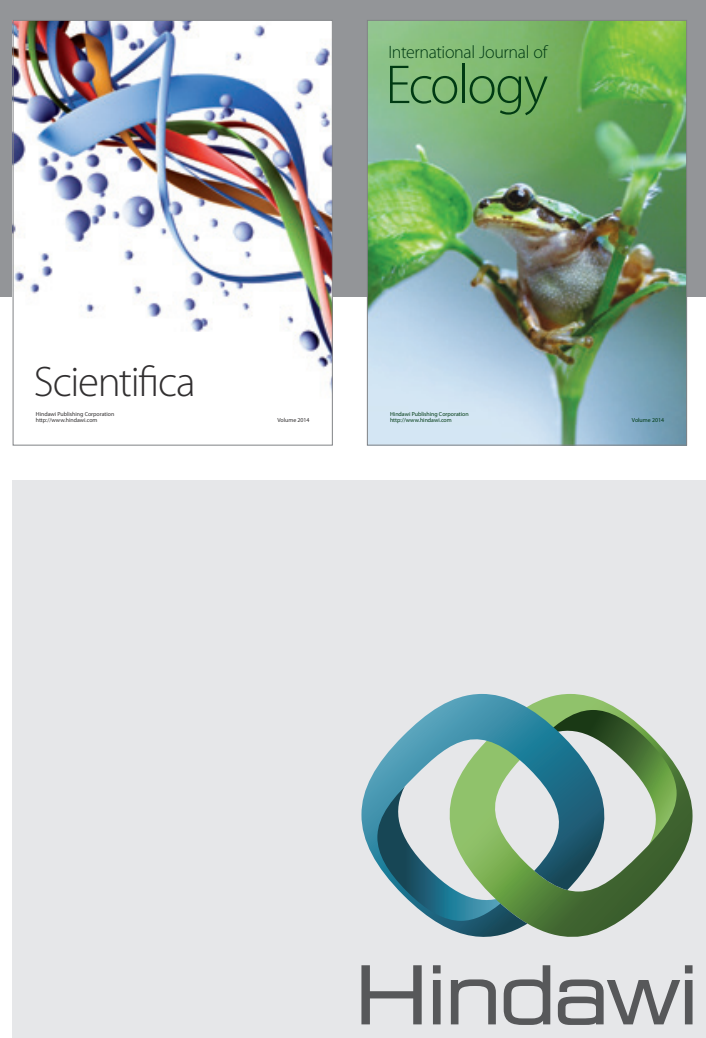

Submit your manuscripts at

http://www.hindawi.com
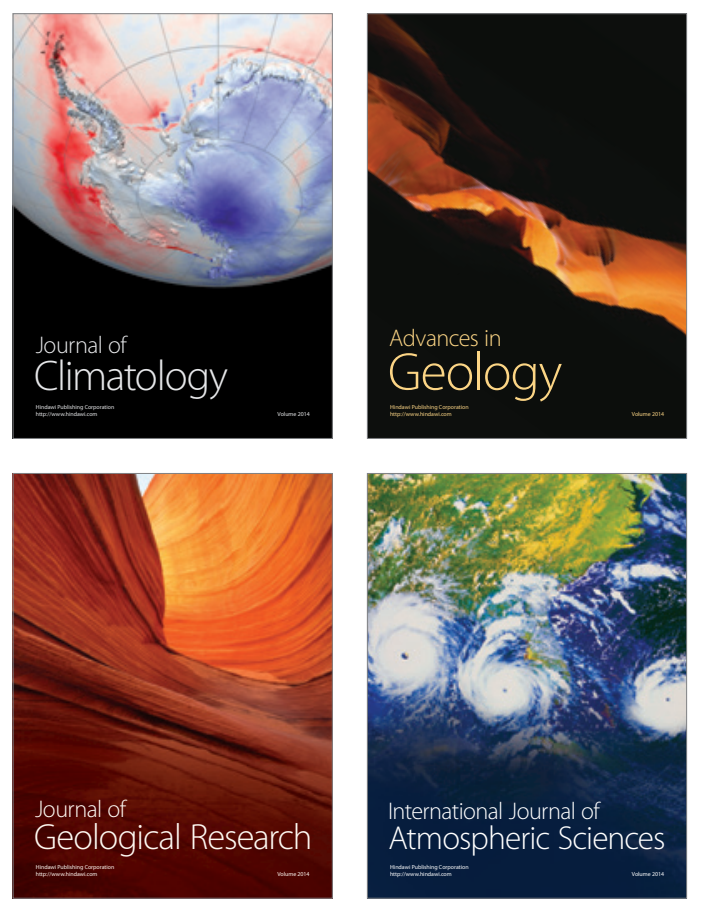

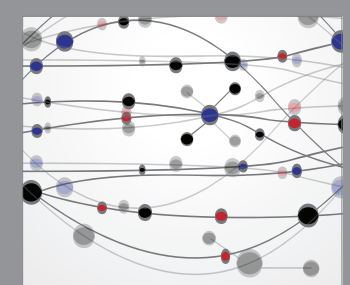

The Scientific

\section{World Journal}
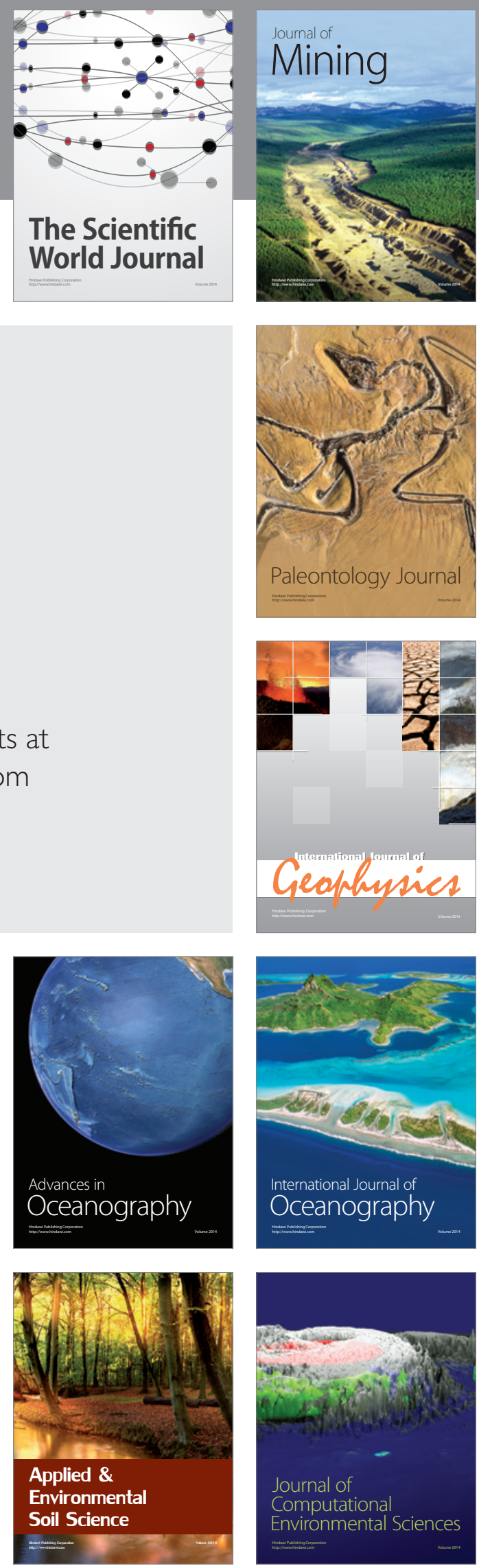